\title{
Comparison of two triiodothyronine uptake techniques for the assessment of thyroid function
}

\author{
VIVIAN CHAN, JOAN MCALISTER, AND J. LANDON \\ From the Departments of Medical Isotopes and Chemical Pathology, St Bartholomew's Hospital, London
}

SYNOPSIS The present study was designed to compare the Thyopac and Triosorb triiodothyronine uptake tests. A normal range was established for the two procedures. Neither test was affected by iodine-containing drugs or contrast media but abnormal results were obtained during pregnancy and in subjects receiving oestrogens. Both tests proved satisfactory in the diagnosis of hypo- and hyperthyroidism, giving results consistent with clinical assessment and with serum protein-bound iodine determinations. The Thyopac procedure was preferred for a number of reasons. It requires less plasma; it is slightly simpler and quicker and it is not time dependent.

In 1957, Hamolsky, Stein, and Freedberg introduced the $T_{3}$ red cell uptake test, designed to reflect (though not measure) the concentration of free thyroid hormones. This test proved extremely valuable; however, the results obtained were influenced by such factors as the haematocrit. Consequently, Mitchell, Harden, and O'Rourke (1960) and Sterling and Tabachnick (1961) used an ion-exchange resin instead of the patients' red cells, and more recently Abbott Laboratories developed the Triosorb kit in which unbound labelled $T_{3}$ is adsorbed onto a resinimpregnated sponge. After initial difficulties this has proved a satisfactory test procedure in our laboratories (Goodhead and McAlister, 1970). Nonetheless, all these procedures have the disadvantage that they alter the equilibrium between bound and free hormone so that the percentage uptake increases with time. Furthermore, as the rate of dissociation is temperature dependent, both temperature and the duration of incubation must be rigidly standardized. Recently Amersham have introduced the Thyopac kit based on the use of a molecular sieve with a pore size such that only the free fraction can enter its matrices. This procedure has the advantage that the equilibrium of the reaction is not disturbed and, therefore, accurate timing of the period of incubation is unnecessary.

The purpose of the present paper is to compare the simplicity, precision, and diagnostic value of the Triosorb and Thyopac $T_{3}$ procedures.

\section{Materials and Methods}

Sera from 263 subjects were tested, of whom 114 were Received for publication 10 June 1971. euthyroid subjects; 58 patients were receiving drugs which may affect thyroxine transport or had recently had an intravenous pyelogram or aortogram; 36 were pregnant women or women receiving oral ovulation inhibitors; 35 were patients with hyperthyroidism, and 20 patients with primary or secondary hypothyroidism. The diagnosis of thyroid dysfunction was based on careful clinical assessment, together with the results of other tests such as thyroid uptake of ${ }^{131} \mathrm{I}$, serum PBI, and total $\mathrm{T}_{4}$ levels and also the clinical response to therapy.

Blood was taken from each subject using a disposable plastic syringe and was immediately placed into a clean glass tube. Serum was separated and stored frozen until used. Each sample was assayed by the Triosorb and Thyopac $T_{3}$ uptake methods, and, for protein-bound iodine, by a Technicon automated procedure (Technicon Symposium, 1964).

TRIOSORB TEST (ABBOTS, CHICAGO)

Bulk kits, each sufficient for 100 tests, were used. They contained resin sponges, ${ }^{125} I-T_{3}$, plastic reaction tubes, plungers, and aspiration tips.

One $\mathrm{ml}$ of serum was pipetted into the reaction tube, followed by approximately $0.9 \mathrm{ml}$ of ${ }^{125} \mathrm{~J}-\mathrm{T}_{3}$ and a resin sponge. The exact time of adding the sponge was noted, and the contents of each tube were mixed thoroughly. Radioactivity was counted in a well-type scintillation counter (Phillips Automatic Gamma Counter) for $\mathbf{4 0}$ seconds (count 1). After incubation at room temperature for exactly 60 minutes, all the supernatant was aspirated, each sponge was washed three times with distilled water, and the tube and sponge were recounted (count 2). 
Results were calculated according to the manufacturer's instructions with an appropriate correction applied for temperature and incubation time where necessary. The results were expressed in terms of a control pooled serum, included in each assay run, which was taken as having a $\mathrm{T}_{3}$ uptake of $30 \%$.

THYOPAC TEST (RADIOCHEMICAL CENTRE, AMERSHAM)

Each kit consisted of 12 vials containing a measured amount of a molecular sieve and ${ }^{125} \mathrm{I}-\mathrm{T}_{3}$ in buffer, together with a vial of standard reference serum.

One hundred $\mu l$ of serum was pipetted into each vial, the contents were mixed for 10-15 minutes on a slowly rotating blood cell mixer, and then allowed to stand. After the resin had settled, $1 \mathrm{ml}$ of the supernatant (containing protein-bound ${ }^{125}\left[-\mathrm{T}_{3}\right.$ ) was counted for 20 seconds. Pooled and reference sera were assayed with each run, the latter being reconstituted 15 minutes before use by the addition of $1 \mathrm{ml}$ of distilled water. Results were calculated according to the manufacturer's instructions and were also expressed in terms of the Triosorb technique. Thus

Thyopac results of pooled serum

Thyopac result of unknown

$$
\times 30=T_{3} \text { uptake (Triosorb). }
$$

TREATMENT OF DATA

The mean $T_{3}$ uptake and the coefficient of variation of the two methods were determined using aliquots of a pooled serum. Precision of the two was compared by calculating the $F$ factor, where
$F=\frac{\text { Variance }(\text { method } 1)}{\text { Variance }(\text { method } 2)}$

The free thyroxine index (FTI) was calculated using the PBI and Triosorb $T_{3}$ uptake results (Clark and Horn, 1965) according to the formula:

FTI $=$ PBI $\times \frac{\text { Triosorb } T_{3} \text { result for unknown }}{30}$

The FTI was also calculated using the PBI and Thyopac $T_{3}$ result, according to the formula:

FTI $=$ PBI $\times \frac{109 \cdot 5}{\text { Thyopac } T_{3} \text { results for unknown }}$

It should be noted that the difference in the two formulae is because, with the Thyopac technique, the result is a measurement of the protein-bound ${ }^{125} \mathrm{I}_{-} \mathrm{T}_{3}$ whilst the Triosorb method measures the unbound ${ }^{125} \mathrm{I}-\mathrm{T}_{3}$ which is adsorbed by the resin.

\section{Results}

Both methods are relatively simple and quick. Using Thyopac kits it is possible to assay 60 test samples, three reference sela, and three pooled sera in three hours. More than four hours are required when the Triosorb procedure is employed, because of the need to count each sample twice, as opposed to once, and the need to wash the resin sponge three times.

In contrast to the Triosorb method, the Thyopac

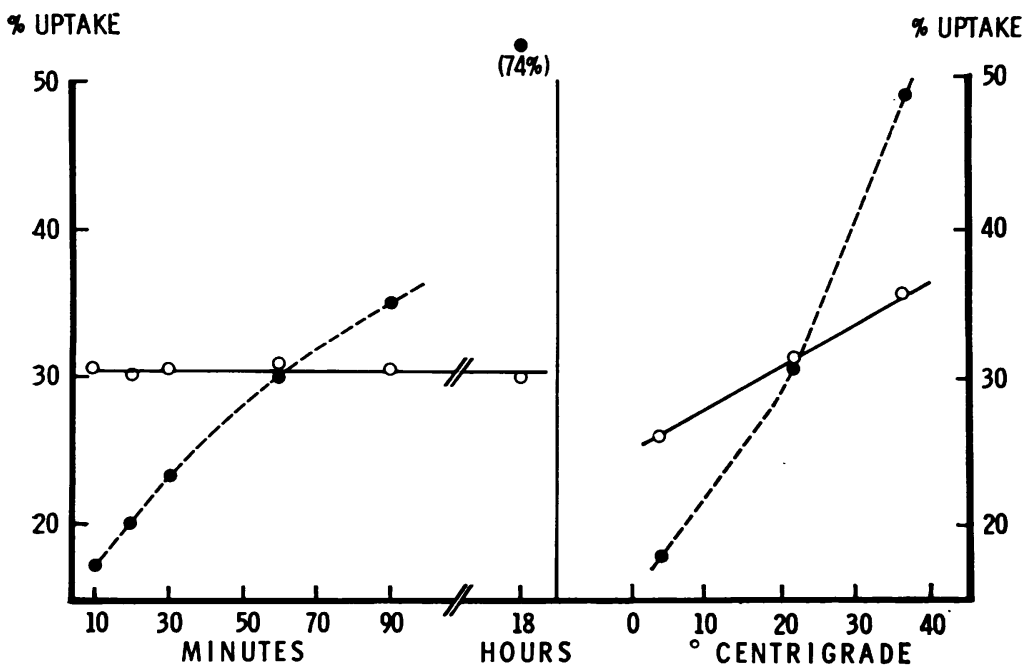

Fig. 1 The effect of the duration and the temperature of incubation on the results obtained using the Thyopac (O_- O) and Triosorb (O.... - . procedures. 
technique is independent of incubation time and less affected by variation in temperature (Fig. 1).

\section{COMPARISON OF 'WITHIN' AND 'BETWEEN' ASSAY PRECISION}

Precision was studied by performing a series of determinations, on each occasion using 10 aliquots of pooled serum. 'Within' assay precision of the Thyopac method $\left(T_{3}\right.$ uptake $=109.9 \pm 1.53$; coefficient of variation $=1.39$ ) is not significantly different from that of the Triosorb technique $\left(T_{3}\right.$ uptake $=32.1 \pm 0.7$; coefficient of variation $=$ $2.08)$. Similarly, there is no significant difference in the 'between' assay precision of the Thyopac $\left(\mathrm{T}_{3}\right.$ uptake 110.5 $\pm 1 \cdot 70$; coefficient of variation 1.54) and Triosorb ( $\mathrm{T}_{3}$ uptake - 31.5 \pm 0.50 ; coefficient of variation $=1.99$ ) procedures.

'Between' assay precision was also studied using serum from 18 normal subjects. Each sample was immediately separated and the serum stored frozen until assayed in quadruplicate, by two workers on different days, using both methods. The results obtained were used to calculate the $F$ value, which was $4 \cdot 1$. Since the critical $F$ value at the $5 \%$ probability level is $2 \cdot 8$, the precision of the Thyopac method in this study is significantly better than that of the Triosorb.

\section{CORRELATION OF PBI AND $\mathrm{T}_{3}$ UPTAKE BY}

THE TWO METHODS

The results obtained for the $T_{3}$ tests and PBI estimations are summarized in the Table. The normal range of $\mathrm{T}_{3}$ uptake obtained using the Thyopac method was $97-122 \%$ with a mean of $109.0 \%$ (Fig. 2). There was no significant difference between the mean $\mathrm{T}_{3}$ value of 24 healthy euthyroid volunteers $(109.7 \%)$ as compared with 90 hospitalized euthyroid subjects $(108.3 \%)$. However, the range of results was greater in the latter group. Using the Triosorb method, normal values ranged from 25 to $35 \%$, with a mean of $30.2 \%$. All serum PBI levels in the control group were within the range 4.0-8.0 $\mu \mathrm{g} \%$. The FTI, calculated using PBI and $\mathrm{T}_{3}$ results obtained by either method, gave the same value of $5 \cdot 8$. Those based on the Thyopac technique are shown in Figure 3.

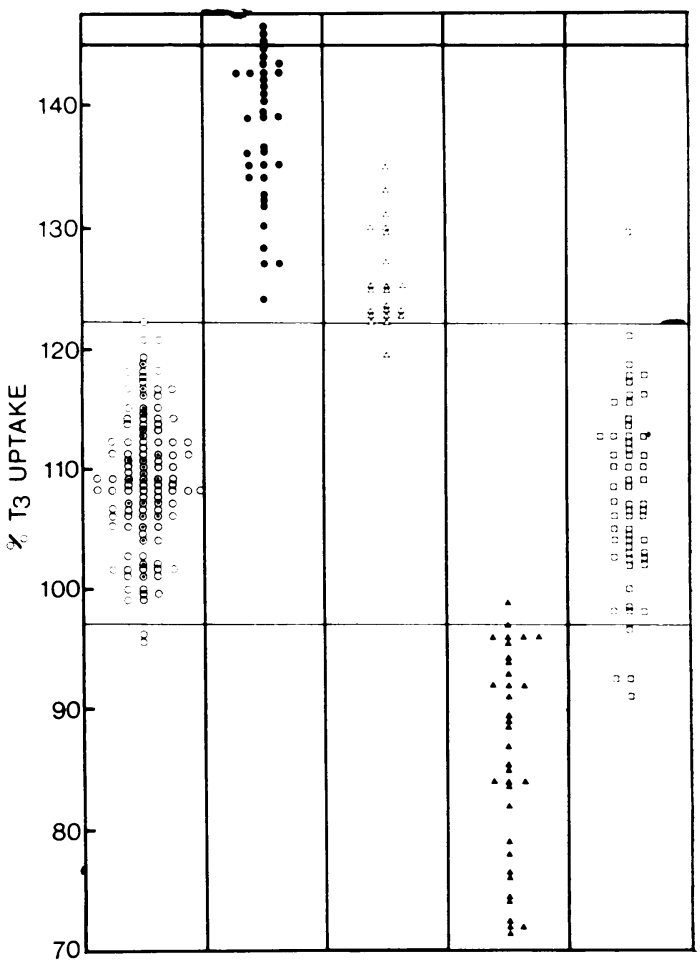

Fig. 2 Thyopac $T_{3}$ uptake in healthy euthyroid subjects $(\bigcirc)$, hospitalized euthyroid subjects $(\bigcirc)$, women who are pregnant or receiving oestrogens (O), patients with primary or secondary hypothyroidism $(\triangle)$, patients with hyperthyroidism $(\mathbf{\Delta})$, and subjects with results possibly affected by drugs etc $(\square)$.

Pregnant women and subjects receiving oestrogen all had raised values as determined by the Thyopac method (mean $138.2 \%$; range $124-148 \%$ ), and a low $\mathrm{T}_{3}$ resin sponge uptake using the Triosorb procedure (mean $19.2 \%$; range $15-25 \%$. Their serum PBI levels ranged from 7 to $16 \mu \mathrm{g} \%$ and all but three had raised levels. The FTI gave a mean value of 5.5 using the Triosorb technique and 6.8 with the Thyopac procedure and, in the great majority, lay in the normal range (Fig. 3).

\begin{tabular}{|c|c|c|c|c|c|c|}
\hline \multirow[t]{2}{*}{ Group } & \multirow{2}{*}{$\begin{array}{l}\text { No. of } \\
\text { Subjects }\end{array}$} & \multirow{2}{*}{$\begin{array}{l}P B I \\
(\mu g \%)\end{array}$} & \multicolumn{2}{|l|}{$T_{3}$ Uptake } & \multicolumn{2}{|c|}{ Free Thyroxine Index } \\
\hline & & & Triosoı $b$ & Thyopac & Triosorb & Thyopac \\
\hline $\begin{array}{l}\text { Euthyroid } \\
\text { Women pregnant or on oral }\end{array}$ & 114 & $5 \cdot 8 \pm 0.75$ & $30 \cdot 2 \pm 2 \cdot 29$ & $109 \cdot 0 \pm 5 \cdot 2$ & $5 \cdot 8 \pm 0.78$ & $5.8 \pm 0.72$ \\
\hline contraceptive & 37 & $8.6 \pm 1.07$ & $19 \cdot 2 \pm 2 \cdot 06$ & $138 \cdot 2 \pm 5 \cdot 80$ & $5.5 \pm 0.99$ & $6 \cdot 8 \pm 1 \cdot 34$ \\
\hline Hyperthyroid & 35 & $11.9 \pm 2.92$ & $42.0 \pm 6.09$ & $86.0 \pm 8.56$ & $16.9 \pm 6.02$ & $15 \cdot 3 \pm 4 \cdot 35$ \\
\hline Hypothyroid & 20 & $1.9 \pm 0.98$ & $22 \cdot 6 \pm 1 \cdot 8$ & $125 \cdot 6 \pm 4 \cdot 32$ & $1.4 \pm 0.73$ & $1.6 \pm 0.88$ \\
\hline
\end{tabular}

Table Summary of PBI, $T_{3}$ uptakes, and FTI results in different groups of subjects 


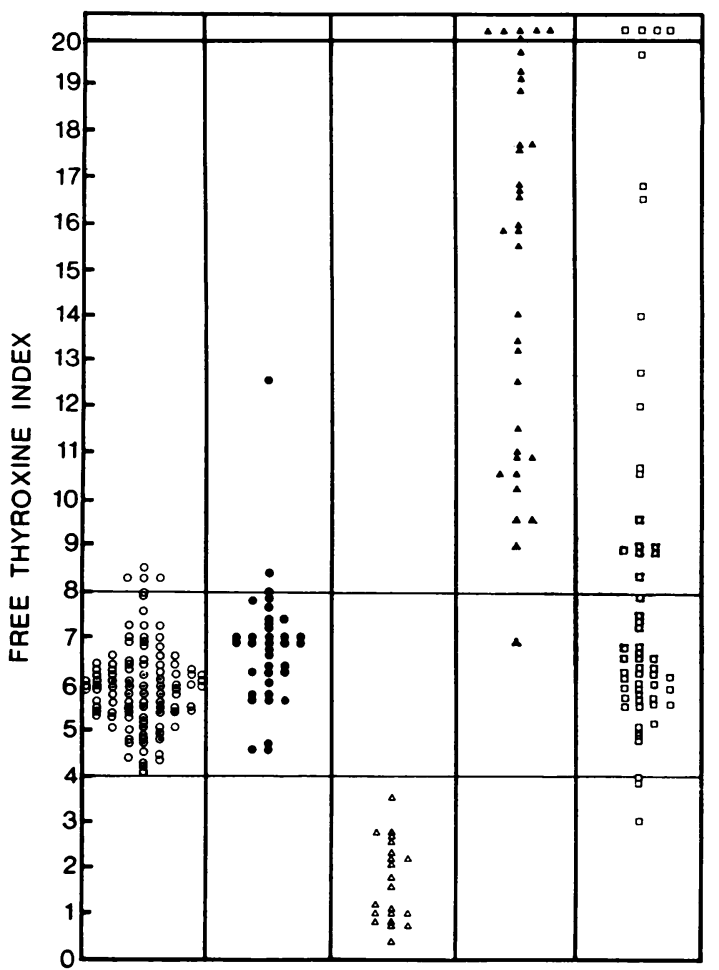

Fig. 3 Free thyroxine index based on the results of $P B I$ and Thyopac $T_{3}$ uptake determinations. Healthy euthyroid subjects $(\odot)$, hospitalized euthyroid subjects $(\bigcirc)$, women who are pregnant or receiving oestrogens (O), patients with primary or secondary hypothyroidism $(\triangle)$, patients with hyperthyroidism $(\Delta)$, and subjects with results possibly affected by drugs etc ( $\square$ ).

All patients with hyperthyroidism had $T_{3}$ values below the normal range using the Thyopac procedure, with the exception of one woman who was receiving an oral ovulation inhibitor. Two of the 35 subjects had a normal Triosorb $T_{3}$ resin sponge uptake and the remainder abnormally high values. Protein-bound iodine levels were raised in all these patients as was their free thyroxine index. The mean FTI calculated using the Thyopac results $(15 \cdot 3)$ was lower than that obtained using Triosorb results (16.9) due to the higher $T_{3}$ values obtained with the latter procedure.

With both $T_{3}$ techniques, one of the 20 hypothyroid patients had a normal value. Protein-bound iodine levels were all below $4.0 \mu \mathrm{g} \%$ and their FTI values were also low, with a mean value of 1.8 and 1.6 as calculated using the Thyopac and Triosorb results respectively.

The PBI was affected in many of the subjects

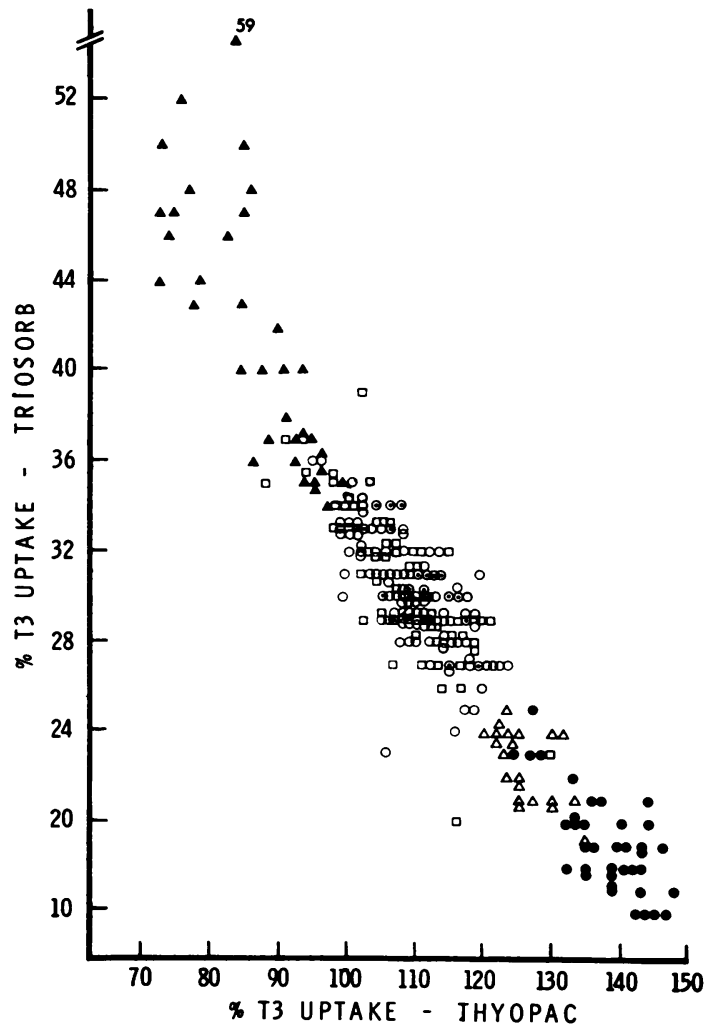

Fig. 4 The correlation of $T_{3}$ uptake determined by the Thyopac and Triosorb procedures. Healthy euthyroids $(\odot)$, hospitalized euthyroids $(\bigcirc)$, women who are pregnant or receiving oestrogens $(\Theta)$, patients with primary or secondary hypothyroidism $(\triangle)$, patients with hyperthyroidism $(\Delta)$, and subjects with results possibly affected by drugs etc ( $\square)$.

receiving iodine-containing contrast media and drugs such as salicylates. The $T_{3}$ tests were affected by the drugs but not by the contrast media.

The correlation of $T_{3}$ values determined by the two methods is shown in Figure 4. There was a negative non-linear relationship, the non-linearity being most marked in the thyrotoxic subjects.

\section{Discussion}

Both the Triosorb and Thyopac methods are relatively simple and rapid to perform. The Thyopac procedure was found to have certain advantages. Only $100 \mu \mathrm{l}$ of serum is required, as compared with $1 \mathrm{ml}$ for the Triosorb. The main advantage is that the Thyopac technique is not time-dependent. This is a distinct advantage in a busy laboratory, especially when the assay is being done by relatively inex- 
perienced personnel. Similar findings on the time dependence of the Thyopac test have been made by Clark and Brown (1970a). We have also found that the technique is less influenced by the temperature of incubation, and no correction is necessary for normal variations in laboratory temperature.

Clark and Brown (1970a) found a coefficient of variation of 4.41 when two sera were tested on different occasions using the Thyopac technique. It is equally precise in our hands. Multiple tests on 18 normal subjects showed a significantly better 'between' assay precision for the Thyopac than for the Triosorb. 'Within' assay precision and 'between' assay precision using the same serum were not significantly different for the two techniques.

In this study the normal range for the Thyopac procedure $(97-122 \%)$ was similar to that reported by Clark in 1969 (90-122\%), while differing somewhat from that suggested by the manufacturers $(90-110 \%)$. The discrepancies are probably due to the different populations studied. Howorth and MacLagan (1969) found that the $T_{3}$ uptake of healthy and hospitalized euthyroid subjects is similar, but that the latter have a greater range. This is in agreement with our findings, but contrary to those of Mitchell et al (1960).

The diagnostic accuracy of the Triosorb method has been proven in our laboratories (Goodhead and McAlister, 1970). Within our somewhat limited experience the diagnostic accuracy of the Thyopac method is equally good. Only one out of 20 patients with hypothyroidism had a normal result, and of 35 hyperthyroid patients only one had a normal $T_{3}$ uptake value. The single exception in the hyperthyroid group was receiving an oral ovulation inhibitor, which is known to affect the $T_{3}$ uptake value.

The Technicon automated method for PBI estimations uses an initial resin step to adsorb free iodine. However, in the presence of excess inorganic iodide, as in subjects who have recently received intravenous injections of iodine-containing contrast media or taken iodine-containing drugs, the amount of resin used is insufficient to adsorb all the free iodide and elevated PBI levels are often found. In the absence of excess iodide the PBI level has been found, in our laboratories, to be a reliable, precise index of thyroid status.

The combination of the PBI and the $T_{3}$ resin uptake (Triosorb) values enables calculation of the FTI and also the free thyroxine factor (FTF) and is particularly useful in pregnant patients and in the ovulem group (Clark and Horn, 1965; Goolden, Gartside, and Sanderson, 1967). However, both our findings and those of Clark and Brown (1970b), that there is a non-linear relationship between the $T_{3^{-}}$ resin uptakes as determined by the two methods, effectively precludes the adaptation of Goolden's FTF (Kintner, 1968) for use with the Thyopacresults. We have, therefore, looked more closely at the FTI in the pregnant and oestrogen-receiving group. Their mean FTI value $(5.5)$ using the Trisorb technique is not significantly different from the mean value of 5.8 obtained for the normal group. This result is contrary to the results of Goolden et al (1967), who used the Triosorb resin sponge, and obtained a highly significant difference between the means of the ovulem and normal groups, and a probably significant difference between the means of the pregnant and normal groups. However, the FTI value $(6 \cdot 8)$ with the Thyopac procedure is significantly greater than the mean value of 5.8 obtained for control subjects, and therefore shows the same trend as their Triosorb results. If the FTI is to be used as a reliable index of thyroid status, it may be necessary to establish a separate euthyroid range of Thyopac FTI for pregnant women and for subjects receiving oestrogens. We are currently investigating this. If the PBI value is in doubt because of suspected iodine contamination, a total $T_{4}$ estimation is helpful, and can be used instead of the PBI to obtain a free thyroxine index.

To summarize, the main advantage of the Thyopac technique is its lack of time dependence. This is because it employs a separation technique which does not disturb the equilibrium between the bound and the free hormone. The non-linear relationship between the Triosorb and Thyopac results, which is particularly marked in the hyperthyroid range, is probably due to further dissociation of the bound fraction when the free hormone is adsorbed onto the resin sponge.

\section{References}

Clark, F. (1969). Technical Bulletin 69/7 on Thyopac-3. Radiochemical Centre, Amersham.

Clark, F., and Brown, H. J. (1970a). Evaluation of Thyopac-3 test in the in-vitro assessment of thyroid function. Brit. med. J., 1, 713-715.

Clark, F., and Brown, H. J. (1970b). Free thyroxine index. Brit. med. J., $2,543$.

Clark, F., and Horn, D. B. (1965). Assessment of thyroid function by the combined use of the serum protein-bound iodine and resin uptake of ${ }^{131}$ I-triiodothyronine. J. clin. Endocr., 25, 39-45.

Goodhead, L., and McAlister, J. M. (1970). An assessment of the triiodothyronine uptake test using a diagnostic kit. In Symposium on In-vitro Procedures with Radioisotopes in Medicine, Vienna, pp. 243-255.

Goolden, A. W. G., Gartside, June M., and Sanderson, C. (1967). Thyroid status in pregnancy and in women taking oral contraceptives. Lancet, 1, 12-15.

Hamolsky, M. W., Stein, M., and Freedberg, A. S. (1957). The thyroid hormone plasma protein complex in man. II. A new in vitro method for study of 'uptake of labelled hormonal components by human erythrocytes'. J. clin. Endocr., 17 33-44.

Howorth, P. J. N., and MacLagan, N. F. (1969). Clinical application of serum-total-thyroxine estimation, resin uptake and freethyroxine index. Lancet, 1, 224-228. 
Kintner, E. P. (1968). Free thyroxine factor and a PBI-T, uptake nonogram. Amer. J. clin. Path., 49, 599-601.

Mitchell, M. L., Harden, A. B., and O'Rourke, M. E. (1960). The in vitro resin sponge uptake of triiodothyrocine- ${ }^{131}$. J. clin. Endocr., 20, 1474-1483.
Sterling, K., and Tabachnick, M. (1961). Resin uptake of ${ }^{121}$ I-Triiodothyronine as a test of thyroid function. J. clin. Endocr., 21, 456-464.

Riley, M., and Goceman, N. (1964). A fully automatic method for the determination of serum protein bound iodine. (Technicon International Symposium, Paper No. 62.) New York. 